\title{
Difference in Relative Telomere Length between Sudanese and Chinese Individuals
}

\author{
Abdelhakam G. Tamomh ${ }^{1,2}$ and Hui Liu ${ }^{1, *}$ \\ ${ }^{1}$ Department of Microbiology and Immunology, College of Medical Laboratory, Dalian Medical University, Dalian 116044, China \\ 2 Department of Parasitology and Medical Entomology, Faculty of Medical Laboratory Sciences, University of El Imam El Mahdi, Kosti, Sudan
}

* Corresponding author: Hui Liu, Department of Microbiology and Immunology, College of Medical Laboratory, Dalian Medical University, Dalian 116044, China. Tel: +8641186110383; Email: liuhui60@sina.com, immunology@dmu.edu.cn

Received 2020 August 07; Revised 2020 August 18; Accepted 2020 September 20.

\section{Abstract}

Background: Racial variations in the relative telomere length have been clearly described or frequently studied. The relative telomere length has emerged as a biological aging marker; however, the difference in the telomere length is debatable between Sudanese and Chinese individuals.

Objectives: The current study investigated the difference in the relative telomere length between Sudanese and Chinese subjects.

Methods: The blood samples of Sudanese and Chinese healthy people were randomly collected, and their deoxyribonucleic acid was obtained in this study. The relative telomere length was measured by the quantitative polymerase chain reaction. The difference in the relative telomere length was analyzed using the Mann-Whitney $U$ test. The degree of distribution in the relative telomere length was assessed by the two-sample Kolmogorov-Smirnov Z test.

Results: According to the obtained results, the difference in the relative telomere length between Sudanese and Chinese individuals was statistically significant $(\mathrm{P}<0.05)$. The frequency of the relative telomere length distributed in Chinese subjects was statistically higher than the frequency reported for the Sudanese subjects $(\mathrm{P}<0.05)$.

Conclusion: A great difference was observed in the relative telomere length between Sudanese and Chinese populations indicating the variation between Sudanese and Chinese races.

Keywords: Environmental factors, Ethnic groups, Genetic factors, Relative telomere length, qPCR
\end{abstract}

\section{Background}

Living in different geographical and environmental areas may contribute or associate with the difference between individuals. The relative telomere length has emerged as a biological aging marker; however, the difference in the telomere length between Sudanese and Chinese individuals is debatable. African (Sudanese) and Asian (Chinese) individuals have different environments, geographical areas, ethnic groups, and genetic factors; therefore, these factors may contribute or associate with the difference between Sudanese and Chinese populations.

The relative telomere length has been described as related to various illness conditions and more affected by environmental and genetic factors (1-4). The telomere length is a bioindicator of cellular aging and maybe an indicator marker for the difference between Sudanese and Chinese individuals. Telomeres are nucleoproteins situated at the ends of all chromosomes in the cell of eukaryotes and participate in the stability and integrity protection and maintenance of the genome during replication (5-7).

The telomere length is a dependable marker and indicator of the vital biological age and replacement for a mitotic clock. Telomeres serve as preventive and protective caps protecting the chromosomal ends from the initiation of apoptosis or senescence (8-11).
Human chromosomal telomeres consist of a short tandem of a (TTAGGG) repeated sequence. The telomere length is a determinant key element of a cell lifespan and closely connected to the explicative age and cell cycle (11-13). Too short telomeres lead to a reduction in the function of cells, thereby contributing to the dysfunction of tissues and organs (14-18).

The length of telomeres decreases by $20-200 \mathrm{bp}$ during a cell division in the majority of the somatic cells in humans. When the shortened length of telomeres is not repaired, it will cause or lead to cell cycle detention and even senescence or apoptosis. The length of telomeres differs and varies between genders and is mostly affected by external environmental factors (19-22). The length of telomeres between individuals can vary at birth and between individuals of the same age $(23,24)$. The length of telomeres can be stated or predicted by the parental length of telomeres, and it is stronger with a paternal effect than a maternal influence $(22,25-27)$.

The role of telomeres has been further described and studied because telomeres have been implicated in numerous diseases, such as cardiovascular diseases, acute kidney injury, and carcinogenesis (2833). In addition, the shortened length of telomeres has been clearly observed in various human diseases, such as aplastic anemia, hepatic disease, and pulmonary fibrosis (34-37). Other diseases are also 
associated with a mutation in genes encoding telomere proteins $(38,39)$.

\section{Objectives}

For the examination or determination of a difference between Sudanese and Chinese populations regarding the presence of the relative telomere length, it is probable to consider the relative telomere length an indicator or index of different benefits to health status among individuals. However, the association of the difference between Sudanese and Chinese populations with the relative telomere length is debatable. Therefore, the present study aimed to examine the relation between the relative telomere length and difference between Sudanese and Chinese healthy individuals.

\section{Methods}

\subsection{Subjects and Specimens}

The present comparative study was carried out on a total of 66 healthy Sudanese $(n=27)$ and Chinese $(n=39)$ individuals with the mean age values of $29.44 \pm 4.07$ and $30.77 \pm 6.9$ years, respectively. The venous blood samples of the healthy individuals were randomly collected. Deoxyribonucleic acid (DNA) was obtained from each sample, and the relative length of telomere was measured among Sudanese and Chinese subjects.

\subsection{Sampling Procedure}

A total of 66 healthy Sudanese and Chinese individuals were included in the study. For all of them, the relative length of telomere was measured by the quantitative polymerase chain reaction (qPCR). In this study, $2 \mathrm{~mL}$ of fresh venous blood was collected from each individual. Then, the blood was added to a container with ethylene diamine tetraacetic acid anticoagulant, and the DNA was extracted based on the manufacture's instructions.

\subsection{Extraction of deoxyribonucleic acid and measurement of relative telomere length}

The blood DNA samples of the Sudanese and Chinese individuals were extracted by a DNA extraction kit (Omega Bio-Tek Inc., Norcross, GA, USA). Afterward, $200 \mu \mathrm{l}$ of blood specimen was used in order to extract genomic DNA. A spectrophotometer (Thermo Fisher, Waltham, MA, USA) was applied to analyze the concentration and determine the purity of DNA which was subsequently stored at $20^{\circ} \mathrm{C}$ in a freezer. The telomere length was analyzed using qPCR to quantify the proportion of the telomere gene to a single copy reference (SCR) gene (T/S). In addition, the $\mathrm{T} / \mathrm{S}$ was commensurable to the relative length of the telomere; therefore, $\mathrm{T} / \mathrm{S}$ was utilized to explain the relative length of the telomere gained from the blood samples of Sudanese and Chinese individuals.

All qPCR procedures were conducted using the Thermal Cycler Dice Real Time System (TaKaRa, Kyoto, Japan). In the present study, qPCR was carried out with the input of $5 \mathrm{ng}$ of DNA, with 32 cycles of the experimental conditions, a predenaturation temperature of $95^{\circ} \mathrm{C}$ for $10 \mathrm{~min}$, denaturation temperature of $95^{\circ} \mathrm{C}$ for $20 \mathrm{sec}$, annealing temperature of $52^{\circ} \mathrm{C}$ for $20 \mathrm{sec}$, and extension temperature of $72^{\circ} \mathrm{C}$ for $45 \mathrm{sec}$. Each of the obtained samples included three parallel samples, and the average measured value was obtained to quantify $\mathrm{T} / \mathrm{S}$ according to the following formula:

$$
\triangle \triangle \mathrm{Cq}=\triangle \mathrm{Cq}(\mathrm{TEL})-\triangle \mathrm{Cq}(\mathrm{SCR}),
$$

Relative $\mathrm{T} / \mathrm{S}(\mathrm{TL})=2^{-\triangle \triangle \mathrm{Cq}}$,

then $\mathrm{TL}=2^{-\triangle \Delta \mathrm{Cq}}$

\subsection{Statistical analysis}

The relative telomere length among the Sudanese and Chinese healthy subjects was presented in quartile values due to the non-normal distribution of the data. The Mann-Whitney U test was utilized for the analysis of the variations between the two populations. The distribution degrees of the relative telomere length between the Sudanese and Chinese individuals were analyzed using the two-sample Kolmogorov-Smirnov Z test. The quantification was carried out by SPSS software (version 21.0). A p-value of $\leq 0.05$ was considered statistically significant.

\subsection{Ethical Consideration}

The present study was approved by the Institutional Ethics Committee of Dalian Medical University, Dalian, China, with the cooperation of the Ethics Committee of Kosti Teaching Hospital, Kosti, Sudan (ethics code: 201805-K.T.H/E.A.L/1/3). In addition, this study was classified as a quality control study, and the need for obtaining patient informed consent was waived.

\section{Results}

Table 1 tabulates the difference in the parameter of the relative telomere length between the Sudanese and Chinese healthy individuals. The relative telomere length in the specimens gained from the African (Sudanese) individuals was significantly lower than the telomere length of the samples collected from the Asian (Chinese) individuals $(\mathrm{P}<0.05)$.

Table 2 and Figure 1 show the variations in the frequency distribution degree of the relative telomere length among the Sudanese and Chinese individuals. The frequency of the measured values of the relative telomere length distributed among the Chinese individuals was statistically higher than that reported for the Sudanese individuals $(\mathrm{P}<0.05)$. The amplification plots and dissociation curves of qPCR among the two Sudanese and Chinese groups were depicted in Figure 2. 
Table 1. Mean difference in relative telomere length between Sudanese and Chinese individuals

\begin{tabular}{|c|c|c|c|c|c|c|c|c|}
\hline \multirow{2}{*}{ Parameter } & \multicolumn{3}{|c|}{ Sudanese } & \multicolumn{3}{|c|}{ Chinese } & \multirow{2}{*}{$\mathbf{Z}$} & \multirow{2}{*}{ P-value } \\
\hline & P25 & P50 & P75 & P25 & P50 & P75 & & \\
\hline Telomere length & 0.216 & 0.253 & 0.371 & 0.306 & 0.412 & 0.669 & -2.850 & 0.004 \\
\hline
\end{tabular}

Table 2. Difference in frequency distribution of relative telomere length between Sudanese and Chinese individuals

\begin{tabular}{lcccc}
\hline Telomere length & Mean & Standard deviation & Kolmogorov-Smirnov Z test & P-value \\
\hline Sudanese & 0.370 & 0.390 & 1.627 & 0.010 \\
Chinese & 0.507 & 0.327 & 1.627 & \\
\hline
\end{tabular}

0.507

B
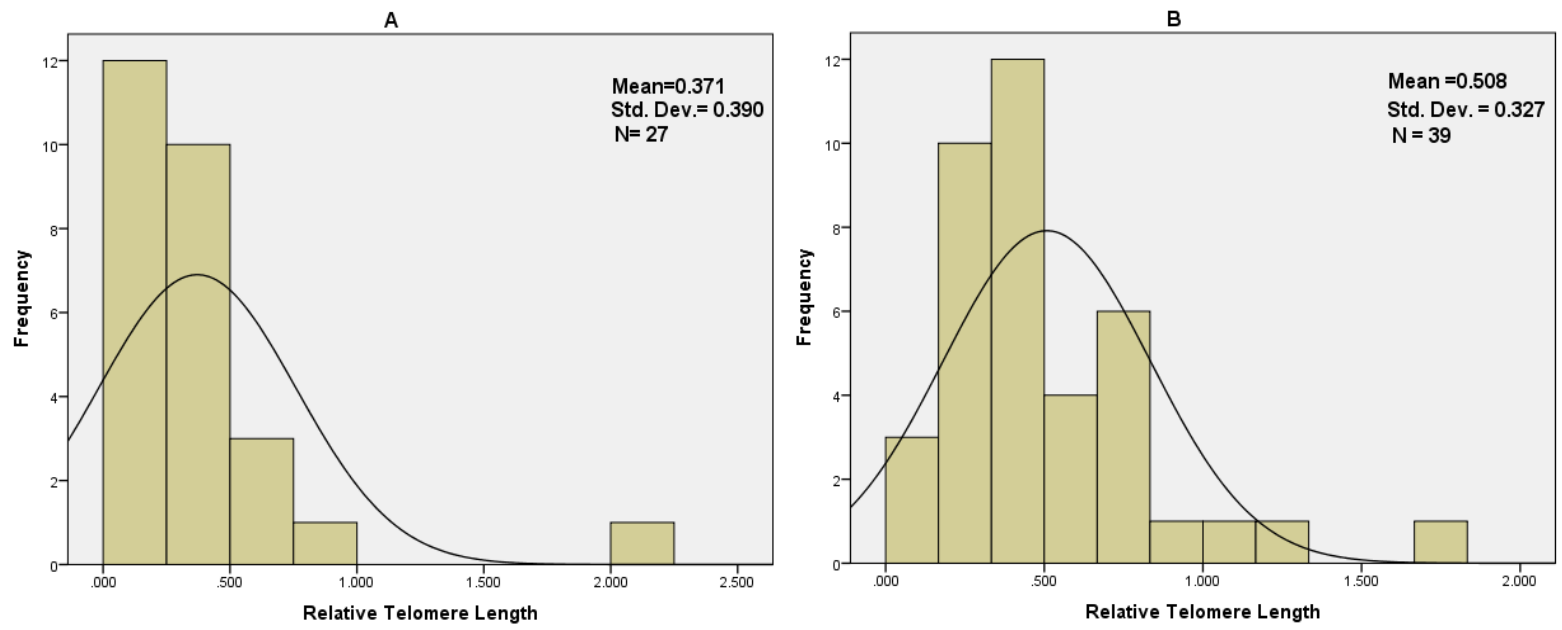

Figure 1. Frequency distribution values of relative telomere length among the two groups; A) Sudanese individuals; B) Chinese individuals
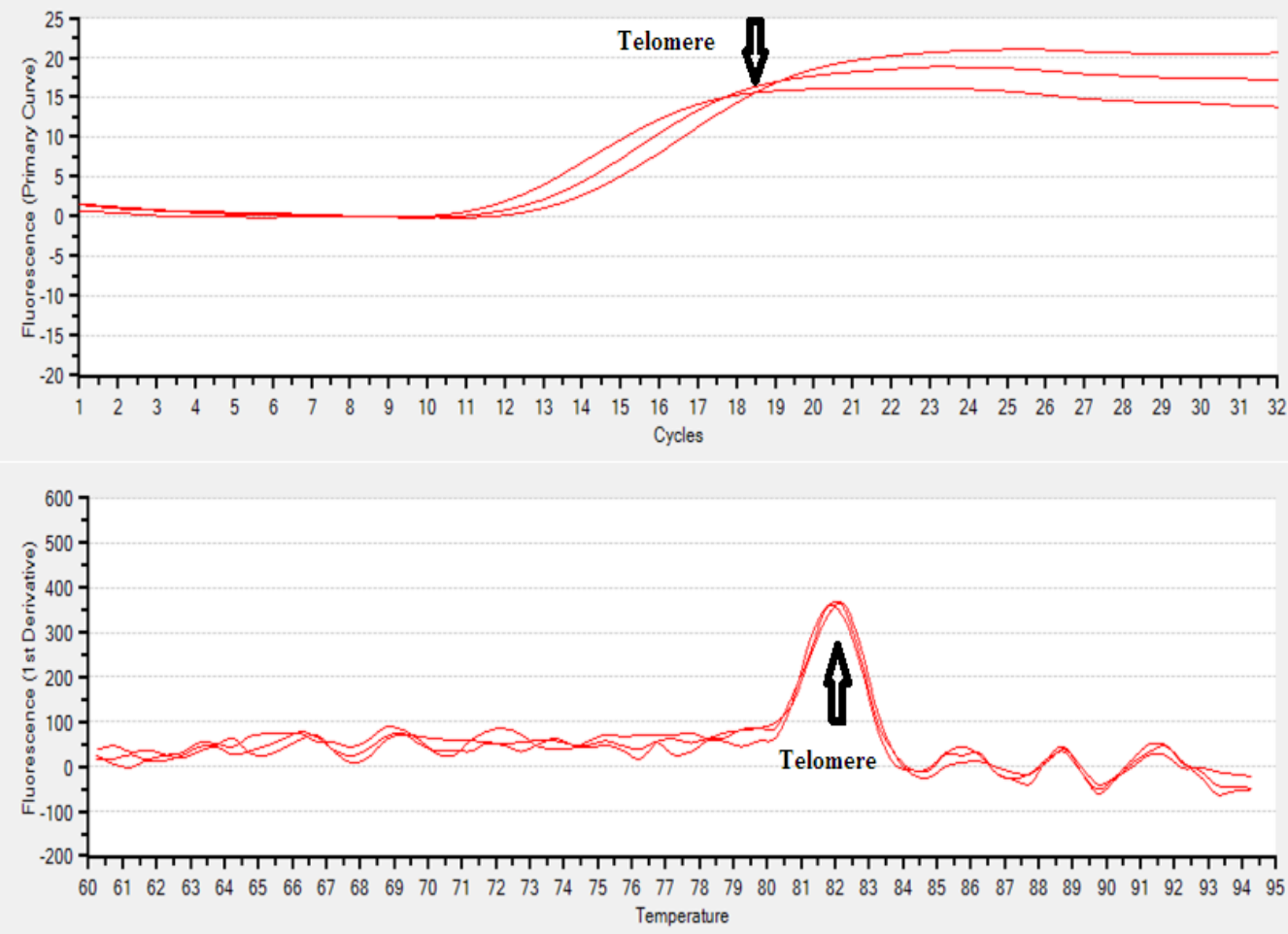

Figure 2. Amplification plots and dissociation curves of quantitative polymerase chain reaction 


\section{Discussion}

The relative telomere length emerged to be a biological indicator of cellular aging. The length of telomeres can differ between individuals and even between individuals of the similar or same age. The Chinese and Sudanese populations more vary in their environmental, geographical, and genetic factors. Therefore, these circumstances or factors may be associated with the difference in the relative telomere length. The values of the relative telomere length measured and analyzed between various ethnic populations, such as Sudanese and Chinese populations, may open new approaches to using the relative telomere length as a marker index of differences between various ethnic groups living in different environments for the evaluation of the health status of individuals.

The relative telomere length has been related to various illness conditions and more affected by environmental and genetic factors $(2,4,6,26,27)$. Most studies describe the relative telomere length among different populations, but not as a marker index of difference between individuals, or even among Sudanese and Chinese individuals as more clearly various ethnic groups. Therefore, the present study aimed to analyze and examine the relative telomere length compare its values between the Sudanese and Chinese individuals and hypothesized it as a marker of difference. The DNA was well extracted from the blood samples of the Sudanese and Chinese healthy individuals, and the relative telomere length was analyzed and calculated using fluorescence qPCR.

The relative telomere length is an essential parameter for describing the cellular aging of individuals and has been rarely applied as a marker of difference to describe or observe the dissimilar characteristic properties of individuals between various ethnic groups, such as between Sudanese and Chinese individuals. Furthermore, the relative telomere length in the current study can predict the variations in the health status of individuals among various populations or provide unique and important information for its function or significance in the difference between individuals.

In the present study, all the included volunteers were healthy subjects from the Chinese and Sudanese populations. The findings of the current study showed that the relative telomere length was significantly higher in the Chinese individuals than that reported for the Sudanese subjects. The length of relative telomere might vary resulting from the variations in external environmental factors or genetic factors of individuals (1-4). The results of the present study indicated an obvious difference in the measured values of the relative telomere length between the Sudanese and Chinese subjects (Table 1).

The results of the two-sample Kolmogorov-
Smirnov Z test demonstrated that the frequency distribution values of the relative telomere length of the Chinese healthy subjects were significantly higher than the frequency distribution values of the Sudanese healthy people (Figure 1 and Table 2). The length of telomeres can differ between individuals and even between individuals of the similar or same age. In addition, the difference in the relative telomere length might be associated with the variations in living environments or genetic factors between individuals $(6,26,27)$.

The results of the present study suggest that the calculated and analyzed relative telomere length of Chinese and Sudanese healthy individuals can be used as a different marker or index for the assessment of the variations in the health status of different populations. Accordingly, the measurement and calculation of the relative telomere length between the two ethnic populations, such as Chinese and Sudanese, could predict the health status of individuals. Furthermore, using or applying the relative telomere length as a marker for the difference between individuals may help with the perception of differences in environmental and genetics factors between ethnic groups and adjustment to local survivals and responses against numerous illnesses, leading to good or better management, diagnosis, treatment, and health status improvement. It is recommended to carry out studies to investigate the relative telomere length as a biomarker and assess the mechanism and role of telomere to perceive the different characteristics of different ethnic populations.

\section{Conclusion}

Based on the results of the present study, it can be concluded that the telomere length of each race has its own characteristics, and the significance of the telomere length requires further investigations. In addition, a great variation in the relative telomere length distribution between Sudanese and Chinese healthy individuals may clearly explain the difference; therefore, it can be used as a marker index of difference for the evaluation of health status between Sudanese and Chinese races or other ethnic groups. It is suggested to carry out further studies to identify the characteristics of the telomere length among other different populations. Additionally, further studies can be performed to determine the association between the telomere length and effect of various diseases among Sudanese and Chinese populations, as two various ethnic populations.

\section{Footnotes}

Authors' Contribution: Study concept and design, acquisition of data, analysis of data, interpretation of data, and drafting the manuscript: Abdelhakam G. 
Tamomh; Critical revision of the manuscript for important intellectual content: Abdelhakam G. Tamomh and Hui Liu; Statistical analysis: Abdelhakam G. Tamomh and Hui Liu; Study supervision: Hui Liu

Conflicts of interest: The authors declare that there is no conflict of interest, including specific, related, and/or affiliated financial interests.

Ethical Approval: The Institutional Ethics Committee of Dalian Medical University classified this study as a quality control study and waived the need for obtaining patient informed consent.

Funding/Support: The present study was not financially supported by any institutions.

\section{References}

1. Campa D, Matarazzi M, Greenhalf W, Bijlsma M, Saum KU, Pasquali $\mathrm{C}$, et al. Genetic determinants of telomere length and risk of pancreatic cancer: A PANDoRA study. Int $J$ Cancer. 2019; 144(6):1275-83. doi: 10.1002/ijc.31928. [PMID: 30325019].

2. Nacopoulos C, Gkouskou K, Karypidis D, Vlastos I, Vesala AM, Choukroun J, et al. Telomere length and genetic variations affecting telomere length as biomarkers for facial regeneration with platelet-rich fibrin based on the low-speed centrifugation concept. J Cosmet Dermatol. 2019;18(1):408-13. doi: 10.1111/ jocd.12666. [PMID: 29761887].

3. Wang S, Chang E, Byanyima P, Huang P, Sanyu I, Musisi E, et al. Association between common telomere length genetic variants and telomere length in an African population and impacts of HIV and TB. J Hum Genet. 2019;64(10):1033-40. doi: 10.1038/s10038-019-0646-9. [PMID: 31388112].

4. Belmaker A, Hallinger KK, Glynn RA, Winkler DW, Haussmann MF. The environmental and genetic determinants of chick telomere length in Tree Swallows (Tachycineta bicolor). Ecol Evol. 2019;9(14):8175-86. doi: 10.1002/ece3.5386. [PMID: 31380080].

5. McKenna MJ, Robinson E, Taylor L, Tompkins C, Cornforth MN, Simon SL, et al. Chromosome translocations, inversions and telomere length for retrospective biodosimetry on exposed U.S. atomic veterans. Radiat Res. 2019;191(4):311-22. doi: 10.1667/rr15240.1. [PMID: 30714852].

6. Song DY, Kim JA, Jeong D, Yun J, Kim SM, Lim K, et al. Telomere length and its correlation with gene mutations in chronic lymphocytic leukemia in a Korean population. PLoS One. 2019;14(7):e0220177. doi: 10.1371/journal.pone.0220177. [PMID: 31335885].

7. Gillis JC, Chang SC, Wang W, Simon NM, Normand SL, Rosner $\mathrm{BA}$, et al. The relation of telomere length at midlife to subsequent 20-year depression trajectories among women. Depress Anxiety. 2019;36(6):565-75. doi: 10.1002/da.22892. [PMID: 30958913].

8. Fan HC, Chen CM, Chi CS, Tsai JD, Chiang KL, Chang YK, et al. Targeting telomerase and ATRX/DAXX inducing tumor senescence and apoptosis in the malignant glioma. Int J Mol Sci. 2019;20(1):200. doi: 10.3390/ijms20010200. [PMID: 30625996].

9. Tsoukalas D, Fragkiadaki P, Docea AO, Alegakis AK, Sarandi E, Vakonaki E, et al. Association of nutraceutical supplements with longer telomere length. Int J Mol Med. 2019;44(1):218-26. doi: 10.3892/ijmm.2019.4191. [PMID: 31115552].

10. Li P, Meng Y, Wang Y, Li J, Lam M, Wang L, et al. Nuclear localization of Desmoplakin and its involvement in telomere maintenance. Int J Biol Sci. 2019;15(11):2350-62. doi: 10.7150/ijbs.34450. [PMID: 31595153].

11. Liu P, Zhang Y, Ma L. Telomere length and associated factors in older adults with hypertension. J Int Med Res. 2019;47(11): 5465-74. doi: 10.1177/0300060519882570. [PMID: 31662013].

12. Liu J, Wang L, Wang Z, Liu JP. Roles of telomere biology in cell senescence, replicative and chronological ageing. Cells. 2019; 8(1):54. doi: 10.3390/cells8010054. [PMID: 30650660].

13. Fali T, Papagno L, Bayard C, Mouloud Y, Boddaert J, Sauce D, et al. New insights into lymphocyte differentiation and aging from telomere length and telomerase activity measurements. Immunol. 2019;202(7):1962-9. doi: 10.4049/jimmunol.1801475. [PMID: 30737273].

14. Okamoto K, Seimiya H. Revisiting telomere shortening in cancer. Cells. 2019;8(2):107. doi: 10.3390/cells8020107. [PMID: 30709063].

15. Amano H, Chaudhury A, Rodriguez-Aguayo C, Lu L, Akhanov V, Catic A, et al. Telomere dysfunction induces Sirtuin repression that drives telomere-dependent disease. Cell Metab. 2019; 29(6):1274-90.e9. doi: 10.1016/j.cmet.2019.03.001. [PMID: 30930169].

16. Chen X, Zeng C, Gong C, Zhang L, Wan Y, Tao F, et al. Associations between early life parent-child separation and shortened telomere length and psychopathological outcomes during adolescence. Psychoneuroendocrinology. 2019;103:195202. doi: 10.1016/j.psyneuen.2019.01.021. [PMID: 30711896].

17. Whittemore K, Vera E, Martínez-Nevado E, Sanpera C, Blasco MA. Telomere shortening rate predicts species life span. Proc Natl Acad Sci U S A. 2019;116(30):15122-7. doi: 10.1073/pnas.1902452116. [PMID: 31285335].

18. Fathi E, Charoudeh HN, Sanaat Z, Farahzadi R. Telomere shortening as a hallmark of stem cell senescence. Stem Cell Investig. 2019;6:7. doi: 10.21037/sci.2019.02.04. [PMID: 31019963].

19. Shin D, Shin J, Lee KW. Effects of inflammation and depression on telomere length in young adults in the United States. Clin Med. 2019;8(5):711. doi: 10.3390/jcm8050711. [PMID: 31109116].

20. Patrick M, Weng NP. Expression and regulation of telomerase in human $\mathrm{T}$ cell differentiation, activation, aging and diseases. Cell Immunol. 2019;345:103989. doi: 10.1016/j.cellimm. 2019.103989. [PMID: 31558266].

21. Kesäniemi J, Lavrinienko A, Tukalenko E, Boratyński Z, Kivisaari K, Mappes T, et al. Exposure to environmental radionuclides associates with tissue-specific impacts on telomerase expression and telomere length. Sci Rep. 2019 9(1):850. doi: 10.1038/s41598-018-37164-8. [PMID: 30696885].

22. Kim JH, Nam CM, Lee D, Bang H, Ko JH, Lim I, et al. Heritability of telomere length across three generations of Korean families. Pediatr Res. 2020;87(6):1060-5. doi: 10.1038/s41390-0190699-7. [PMID: 31783399].

23. Huang Y, Dai W, Li Y. Potential associations of testosterone/estradiol ratio, leukocyte hTERT expression and PBMC telomerase activity with aging and the presence of coronary artery disease in men. Exp Gerontol. 2019;117:38-44. doi: 10.1016/j.exger.2018.08.008. [PMID: 30179663].

24. Kalungi A, Womersley JS, Kinyanda E, Joloba ML, Ssembajjwe W, Nsubuga RN, et al. Internalizing mental disorders and accelerated cellular aging among perinatally HIV-infected youth in uganda. Front Genet. 2019;10:705. doi: 10.3389/ fgene.2019.00705. [PMID: 31428136].

25. Lazarides C, Epel ES, Lin J, Blackburn EH, Voelkle MC, Buss C, et al. Maternal pro-inflammatory state during pregnancy and newborn leukocyte telomere length: a prospective investigation. Brain Behav Immun. 2019;80:419-26. doi: 10.1016/j.bbi.2019.04.021. [PMID: 30974172].

26. Song L, Zhang B, Liu B, Wu M, Zhang L, Wang L, et al. Effects of maternal exposure to ambient air pollution on newborn telomere length. Environ Int. 2019;128:254-60. doi: 10.1016/j.envint.2019.04.064. [PMID: 31059920].

27. Eisenberg DT, Rej PH, Duazo P, Carba D, Hayes MG, Kuzawa CW. Testing for paternal influences on offspring telomere length in a human cohort in the Philippines. Am J Phys Anthropol. 2020;171(3):520-8. doi: 10.1002/ajpa.23983. [PMID: 31845317].

28. Koriath M, Müller C, Pfeiffer N, Nickels S, Beutel M, Schmidtmann I, et al. Relative telomere length and cardiovascular risk factors. Biomolecules. 2019;9(5):195. doi: 10.3390/biom9050192. [PMID: 31108918].

29. Xu X, Hu H, Lin Y, Huang F, Ji H, Li Y, et al. Differences in 
leukocyte telomere length between coronary heart disease and normal population: a multipopulation meta-analysis. Biomed Res Int. 2019;2019:5046867. doi: 10.1155/2019/5046867. [PMID: 31198785].

30. Pusceddu I, Herrmann W, Kleber ME, Scharnagl H, Hoffmann MM, Winklhofer-Roob BM, et al. Subclinical inflammation, telomere shortening, homocysteine, vitamin B6, and mortality: the Ludwigshafen risk and cardiovascular health Study. Eur J Nutr. 2020;59(4):1399-411. doi: 10.1007/s00394-019-019938. [PMID: 31129702].

31. Cokan Vujkovac A, Novaković S, Vujkovac B, Števanec M, Škerl P, Šabovič M. Aging in Fabry disease: role of telomere length, telomerase activity, and kidney disease. Nephron. 2020; 144(1):5-13. doi: 10.1159/000502909. [PMID: 31509825].

32. Chen X, Wei S, Ma H, Jin G, Hu Z, Suping H, et al. Telomere length in cervical exfoliated cells, interaction with HPV genotype, and cervical cancer occurrence among high-risk HPV-positive women. Cancer Med. 2019;8(10):4845-51. doi: 10.1002/cam4.2246. [PMID: 31243901].

33. Srinivas N, Rachakonda S, Hielscher T, Calderazzo S, Rudnai P, Gurzau E, et al. Telomere length, arsenic exposure and risk of basal cell carcinoma of skin. Carcinogenesis. 2019;40(6):71523. doi: 10.1093/carcin/bgz059. [PMID: 30874287].

34. Arias-Salgado EG, Galvez E, Planas-Cerezales L, PintadoBerninches L, Vallespin E, Martinez P, et al., Genetic analyses of aplastic anemia and idiopathic pulmonary fibrosis patients with short telomeres, possible implication of DNArepair genes. Orphanet J Rare Dis. 2019;14(1):82. doi: 10.1186/s13023-019-1046-0. [PMID: 30995915].

35. Shen W, Kerr CM, Przychozen B, Mahfouz RZ, LaFramboise T, Nagata $\mathrm{Y}$, et al. Impact of germline CTC1 alterations on telomere length in acquired bone marrow failure. $\mathrm{Br} J$ Haematol. 2019;185(5):935-9. doi: 10.1111/bjh.15862. [PMID: 30891747].

36. Demerdash HM, Elyamany AS, Arida E. Impact of direct-acting antivirals on leukocytic DNA telomere length in hepatitis C virus-related hepatic cirrhosis. Eur J Gastroenterol Hepatol. 2019;31(4):494-8. doi: 10.1097/meg.0000000000001306. [PMID: 30444746].

37. Arish N, Petukhov D, Wallach-Dayan SB. The role of telomerase and telomeres in interstitial lung diseases: from molecules to clinical implications. Int J Mol Sci. 2019;20(12):2996. doi: 10.3390/ijms20122996. [PMID: 31248154].

38. Borie R, Bouvry D, Cottin V, Gauvain C, Cazes A, Debray MP, et al. Regulator of telomere length 1 (RTEL1) mutations are associated with heterogeneous pulmonary and extrapulmonary phenotypes. Eur Respir J. 2019;53(2):1800508. doi: 10.1183/13993003.00508-2018. [PMID: 30523160].

39. Ventura A, Pellegrini C, Cardelli L, Rocco T, Ciciarelli V, Peris K, et al. Telomeres and telomerase in cutaneous squamous cell carcinoma. Int J Mol Sci. 2019;20(6):1333. doi: 10.3390/ ijms20061333. [PMID: 30884806]. 